\title{
PETRUS POSSINUS
}

\section{$\begin{array}{llllllll}\mathbf{L} & \mathbf{E} & \mathbf{C} & \mathbf{T} & \mathbf{O} & \mathbf{R} & \mathbf{I} \text {. }\end{array}$}

Quod nunc primum e Graeciae naufragiis emergit Georgius Pachymeres, historicus non minus alioqui luce dignus Latina quam multi eius aequales ac posteri iam pridem typis et literis evulgati nostratibus, non sola infelicitas sed culpa eius quaedam fecit. retrusus et antiquarius scriptor, quem loqui cum vulgo quidquam piget, praeterea perplexus et hiantis sententiae compositor, tot tenebris suas vigilias obvolvit, ut eruditissimorum, quibus innotuit, interpretum a sui editione tentanda curas conatusque deterruerit; idem passus quod sepias aiunt atque torpedines, qui pisces quod rarius mensarum honore dignenlur, caligini quam offundunt, torpori quem afflant, imputent, illa oculos, hoc manus et brachia piscantium a nisu eos e nativo gurgile tollendi soliti repellere.

II. Erat tamen haud dubium operae pretium in eius publicatione historiae, quae res magnas ac memorabiles per annos fere octoginta saeculi obscuri ac testium inopis gestas, si minus semper diserta, saltem prudenti diligenti morata et plerumque fideli narratione prosequatur; quae postremae quasi scenae Orientalis imperii sub domo Palaeologa ita principia exhibet, ut in is causas tragicae catastrophes, qua Turcis et Muhammedi Constantini regia succubuit, advertere prudens lector manifestas queat; quae plurima docet nusquam alibi notata; quae a Niceta et Acropolita ad Cantacuzenum, hiatum quendam explens publicae memoriae, viam quasi regiam insternit albis velut quadrigis historicae veritatis, qua nostra usque ad tempora nuspiam haerente aut abrupta triumpli pompa invehatur; quae denique negotium omnis aevi maximum et cele- 
berrimum, conciliationis ecclesiarum Graecae cum Latina, sic a primis originibus evolvit, sic a deliberationum arcanis verisque rationibus, per anfractus inde fucosarum artium hinc fidei sincerae, ad suos exitus perducit, ut asserere non dubitem id argumentum ingens a nullo plus Graeco scriptore lucis accipere.

III. Non me fugit intercedi adversus haec speciosa et plausibili exceptione posse a merito querentibus Pachymerianam hanc historiam non raro Graecorum scbismali favere, Latinorum res et iura deterere narrando, auctoris pronum in partem sequiorem multis modis studium ostendere. non nego, non excuso; et ita factum, et large in hoc peccatum a nostro confiteor. tamen contendo utilem publico et ipsi quinetiam in his laesae veritati atque orthodoxae fidei optabilem talis libri evulgationem esse. neque ad hoc evincendum utar praeiudiciis ecclesiae, historias Eusebii Caesariensis, Ariani a multis habiti; Socratis et Sozomeni, palam Novatianorum; Nicephori Callisti e faece licet interdum haurientis haeresum, et alias suspectorum pariter scriptorum, legi sinentis et probantis. aio, vel si omnes quos nominavi exterminarentur; peculiari nomine interesse Apostolicae sedis et Romanae communionis universae, subiici oculis et teri manibus cunctorum hoc $\mathrm{Pa}$ chymeris opus, quoniam in eo sanitas doctrinae de processione Spiritus Sancti ex patre filioque, de primatu Papae et iure provocationis ad Romanam sedem ex quocumque patriarchatu; sincera caritas et vera sanctimonia Gregorii $\mathbf{X}$ aliorumque Romanorum per ea tempora pontificum; Ioannis Vecci Constantinopolitani patriarchae, partes Latinorum studiosissime amplexi, constantissime tuili, eruditio maxima, liberrimum idemque perspicacissimum ingenium, veracitas intrepida, vita inculpata, mores sancti, divinis etiam illustrationibus probati, palan magnificeque praedicantur, idque ab oculato teste, et quod caput est, domo adversantium producto, ipsoque haud dissimulanter adversariis studente, ut quod dicit nobis favens, expressum evidentia veritatis, quod contrarium immiscet, educationis vitio inolitum, subornatum adulatione, ambiliosis spebus, obnoxiis a vi tyrannica timoribus contra officii conscientiam extorlum, merito debeat intelligi, ausim dicere: si to- 
tus orthodoxus esset Pachymeres, minus iuvaret, sunt veneno carentes et prorsus innoxii serpentes: sed ii theriacam non faciunt. viperis opus est exitiali succo turgidis, lethali dente infestis, unde salutare alexipharmacum possit existerc. adeo malis interdum etiam et pestibus sua inest utilitas! habes cur non tolerabilis solum, sed etiam expetibilis schismatici scriptoris edilio sit. caeterum etsi non magis periculum esse video ne Catholicus e Pachymeris lectione schisma discát, quam ne Christianus, deorum falsorum passim occurrente in libris Veterum Graecis Latinisque vulgo tritis mentione, in idololatriam pertrahatur, dedi tamen diligentem operam in Notis ad singula loca maculosa, ne calumniae ac mendacia impune auctori, fraudi lectori forent.

IV. Quod superest, editionis huius consilia causas facultates totamque rationem edisserenti operam mihi ne, quaeso, gravere, lector, brevem commodare. sanctae memoriae URBANUS pontifex eius nominis Octavus duo historica Pachymeris opera, totidem distinctis transmarinae ac perantiquae manus exarata voluminibus, in suo thesauro librario servabat, integra et haud paulo saniora quam quae Augustanus Wolfio visus codex babuit. prior tomus in libros tributus sex Michaëlis Palaeologi res gestas ante imperium et in imperio enarrat, alter libris septem magnam partem actorum longi principatus Andronici Senioris exhibet. utrumque illum a se perlectum commentarium, pro ea qua valebat Graecae linguae peritia doctissimus is pontifex, dignum qui Latine legeretur iudicabat; idque ut procuraret familiarissimo et fidissimo sibi capiti, Eminentissimo Cardinali FRANCISCO BARBERINO Fratris Filio, saepe impenseque commendarit. quam ad hoc ille per hos annos viginti, et quod excurrit, diligentiam adhibuerit, infinitum sit minute persequi. satis fuerit dixisse nihil in hoc genere quod utcumque tenlari posset, ab eo unquarn uspiamve praetermissum. obtulit, invitavit, rogavit, promisit, voce coram, scriptis literis, per amicos conventis viris doctis : nihil denique non egit, quo Pachymeri Lativi alicunde inlerpretis suppeteret copia, missis etiam procul extra Italiam ipsis codicilus archetypis, quasi ut suam per se ipsi causam agerent et miserationem praesentes ac supplices moverent; 
quos tali occasione multis annis in viri doctissimi iacuisse scriniis multi sciunt. successus fuit, qualem comitiorum de regno Arborum sacer apologus memorat, ut nimirum vite, oliva, ficu, cunctis pro se quaque plantis felicibus recusantibus, ad rhamnum res derolveretur. licét eram mihi conscius suppelfectilis curtae, minoris facultatis, temporis diversissimis occupationibus addictissimi, tamen cognitis desideriis viri summi de me optime meriti, verecundiae meae vim facientibus et imperiorum occupantibus partes, audere supra vires non dubitavi, eiusque onus negotii subire, in quo sustinendo defungendogue vice otii ingeniique omnis studium mihi esse deberet atque obsequium.

V. Sic ad eum laborem aggrediens, in recensendo vertendoque fundum habui Barberinum codicem, eum de quo multa dixi, omnium, opinor, qui extant uspiam integerrimusn et optimum, in charta transmarina, vetustatis auctori Pachymeri aequalis, manus non speciosissimae quidem aut lectu facillimae, caeterum eruditae et exactae. duos consului alios in dubiis, Vaticanum et Allatianum. priori a bibliotheca Pontificia, ubi asservatur, nomen feci. fuit is quondam V. C. Aloysii Lollini episeopi Bellunensis, gui eum moriens cum aliis mss, quae multa collegerat, Vaticanae bibliothecae legavit. hunc ut inde domum auferrem, ibique ad continuum et liberiorem usum multis mensibus tenerem $S$. D. N. ALEXANDRI VII praestans benignitas concessit. tertium humanissime suppeditavit e privato peculio librario Illustrissimus Leo Allatius praefectus bibliothecae Vaticanae. praefert is antiquitatem quadringentorum facile annorum chartae et scriptu-

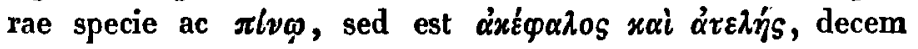
circiter capitibus primis mutilus, finem item lacunis interpellatum et iniuriis aevi ac tempestatum corruptum habens. Vaticanus longe recentioris est manus. in Barberino codice pertusis casu quodam foliis duobus, in capitibus 5 et 6 libri $I$ hiatus totiden fuere, quos explere non potui ex Allatiano, qui primis, ut dixi, capitibus decem caret, nec ex Vaticano, qui non tam apographum historiae Pachymeris quam compendium est, diversis pleraque verbis exprimens, non paucis praetermissis. recurri ergo ad exemplar asservatum in bibliotheca 
regis Christianissimi Parisiensi, unde meo rogatu R. P. Philippus Labbe, quod opus erat ambabus lacunis coaequandis, diligenter exscripsit et misit.

VI. Interpretationis non anxie addictae, sed et in paraphrasim aliquando redundantis haec peculiaris excusatio est in hoc omnium, qui Graece historias ediderunt, obscurissimo scriptore. lectorum alii Graece sciunt, alii (quae maior turba est) non item. neutrorum accommodatam rationibus putabam versionem scrupulose sedulam, balbutientem et illautam, quae ieiuna squallidis, intricata perplexis, ambigua confusioribus mularet. nam Graece quidem docti, si queis cupido insit indolem noscendi Pachymerianae dictionis, longe id assequentur facilius Graeco ipso contextu protinus visendo, quam male terso intuendo velut quodam speculo interpretamenti sesquibarbari, latinitatem invitissimam per omnia graecismi vestigia lutulento tramite raptantis. caeterorum qui una freti Latinae linguae peritia historiam hanc in manus sument, manifestum est non id votum aut destinatum esse, noctium et labyrinthorum ambagiosi scripti experimentum praesens capere, potius quam absconditae defossaeque illic fidei gestarum vere rerum liquido lucidoque testimonio doceri. studui ergo, quo his obsecundarem, indagatum magno plerumque labore meo huius scriptoris sensum quam clarissime proponere, adeogue, ubi res posceret, distincta e confusis, ex horridis nitida, plana e pravis, aperta ex aenigmaticis reddere.

VII. Erunt tamen (scio) quibus nec factum nec facti defensio probabitur. hi vero vel ostracismo me, licet, exterminent, modo testae causam adscribant: proscriptum te volumus, quod tenebricosissimum scriptorem primo aspectu intelligibilem feceris. sed meliora Venusinus auguretur, e diverso responsans

solventur risu tabulae, th missus abibis.

enimvero si mentem scripti non asseguitur, si affingit aliena, si nou repraesentat quidquid subest, iure vapulet interpres: donec quidem latentia producit, involuta exerit, obruta eruit, dum nucleum, succum intimum, meram medullam sententiae abstrusae fracto extractam cortici, velut cibi cocti et conditi paratum legenti ferculum apponit, sinant isti morosi serviri 
suis commodis, et vereantur id damnare unde, nisi dissimulant, se quoque ipsos iuvari sentiunt. nec eo tamen haec trahi velim quasi nuspiam oflendisse me arroganter asseverem, aut ubi meruerim, culpari abnuam. unum deprecor, ne iudicium praecipitetur, neve ad primam naevi speciem veru ac nigrum theta plagosus censor expediat. cognoscere prius ne gravetur in Glossario et Notis, quae me ad sic interpretandum causae impulerint. vel absolvet, vel ignoscet, vel mitius forte castigabit perspecto quid in re sit. typographi menda ne imputentur mihi cur postulem, quod ipsa ultro aequitas largitur? ne multa graviaque intervenirent, cura est non mediocri certatum: ne ulla vel levia irreperent vitare ultra humanam facultatem fuit. index praecipuorum appensus ad calcem $\mathrm{Ob}-$ servationum est, cuius admonitu manu adiuvet qui adverterit, et benefacti locum occupet. chronologicas controversias (nam erant et hic nodi eins generis digni vindice) in librum proprium seposuimus, cui Ė $\pi i \mu \varepsilon \tau \rho o \nu$ superiectum est Specimen sapientiae Indorum veterum ${ }^{*}$ ). liber tertio succedentium invicem translationum gradu ex Indico Persico Arabico Graecus factus, et velut tunc notissimus Constantinopoli, ab imperatore ibi apud hunc nostrum concionante laudatus, quaerendi sui necessitatem imposuit haud aliter intellecturis locum in quo memoratur. inventus in locuplete talium penu, museo Illustrissimi Allatii, eius henigna gratia visus a me Latineque redditus, eiusdem iudicio et hortatu editus hic integer est, non invenusta, ni fallor, peregrinitate placiturus. vale.

*) Eum libellum (Hitopadesa barbare rocatur) nec tum fuisse incognitum et deinde plurimas in linguas conversum vulgo legi ostendunt Careius (Hitopadesa or salutary instruction in the original Sanscrit, printed at Serampore $p$. IY et X) et Iacobus Grimmius (Reinhart Fuchs p. CCIXXIII sqq.). nihil igitur causae erat cur diutius adhaereret libro cum sua mole gravi tum a Possino benignius quam oportebat ornato.

I. B. 